\title{
Uso de bioestimulante na reversão de injúria de glyphosate no milho convencional
}

\author{
Use of biostimulant in the reversion of injury caused by glyphosate on \\ conventional corn plants
}

Christiano Lima Lobo de Andrade ${ }^{1}$, Marcos Paulo Carvalho², Alberto Leão Lemos Barroso ${ }^{2}$, Márcio Rosa², Tulio Porto Gonçalo ${ }^{2}$, Cristiano Buchling 2 , Rafael Lopes Santos Rodrigues ${ }^{3}$

Resumo - O cultivo de milho convencional vem retomando espaço no panorama agrícola nacional em função da dificuldade de controle do milho tiguera em culturas subsequentes. Entretanto, a deriva de glyphosate em lavouras adjacentes passou a ser uma preocupação entre os produtores e técnicos. Dessa forma o experimento foi instalado para avaliar o efeito de baixas doses de glyphosate na cultura e a capacidade de um bioestimulante a base de hormônios vegetais em reverter as injúrias causadas pelo herbicida, avaliando-se características morfológicas e taxa fotossintética de plantas de milho. Foi utilizado o delineamento inteiramente casualizados com quatro repetições em arranjo fatorial 5 X 2 , sendo que o primeiro fator correspondeu a cinco doses de glyphosate $\left(0 ; 10 ; 20 ; 30\right.$ e $40 \mathrm{~g} \mathrm{e}^{\mathrm{a}}$. ha-1 $)$ e o segundo a ausência e presença do bioestimulante. As características avaliadas foram fitointoxicação, diâmetro do colmo, altura plantas e massa fresca de raiz e parte aérea, índice SPAD e taxa fotossintética. A maior dose de glyphosate provocou maior fitointoxicação, além de plantas com menor altura e menor índice SPAD. O bioestimulante proporcionou acréscimos no diâmetro de colmo, índice SPAD e taxa fotossintética. A presença de bioestimulante não proporcionou efeito reparador nos efeitos deletérios provocados pelo glyphosate em plantas de milho convencional.

Palavras-chave: herbicida, fitointoxicação, Zea mays

\begin{abstract}
Conventional corn cultivation has been taking up space in the national agricultural panorama due to the difficulty of controlling the corn in subsequent crops. However, glyphosate drift in adjacent crops has become a concern among producers and technicians. Thus the experiment was installed to evaluate the effect of low doses of glyphosate on the crop and the ability of a plant hormone-based biostimulant to reverse the injuries caused by the herbicide, evaluating the morphological characteristics and photosynthetic rate of corn plants. It was used a completely randomized design with four replicates in factorial arrangement $5 \mathrm{X} 2$, with the first factor corresponding to five doses of glyphosate $\left(0,10,20,30\right.$ and $40 \mathrm{~g}$ e.a ha $\left.{ }^{-1}\right)$, and the second, the absence and presence of the biostimulant. The evaluated characteristics were phytotoxification,

Recebido: Janeiro 12, 2018. Aceito: Outubro 19, 2018

${ }^{1}$ Programa de Pós-graduação em Ciências Agrárias, Instituto Federal Goiano - IF Goiano, Rodovia Sul Goiana, Km 01, Zona Rural, CEP 75906-896, Rio Verde, GO, Brasil. E-mail: christiano.instrutoria@gmail.com

2 Programa de Pós-Graduação em Produção Vegetal, Universidade de Rio Verde - UNIRV, Rio Verde, GO, Brasil.E-mail: marcos_agrofesurv@hotmail.com; albarroso60@gmail.com; marcio1506-@hotmail.com; tulio.goncalo@gapescna.com.br; cristiano.buchling@monsanto.com

${ }^{3}$ Faculdade de Agronomia, Universidade de Rio Verde - UNIRV, Rio Verde, GO, Brasil.

E-mail: rafaellopes1015@gmail.com
\end{abstract}


stalk diameter, plant height and fresh root and shoot mass, SPAD index and photosynthetic rate. The higher dose of glyphosate resulted in higher phytointoxication, plants with lower height and lower SPAD index. Biostimulant provided increases in stalk diameter, SPAD index and photosynthetic rate. The presence of biostimulant did not provide a repairing effect on the deleterious effects caused by glyphosate in conventional corn plants

Keywords: herbicide, phytotoxification, Zea mays

\section{Introdução}

O milho (Zea mays) é considerado uma commodity agrícola apresentando grande importância no mercado agrícola mundial por estar elencado na base alimentar humana além de amplamente utilizado na alimentação animal (Ribas, 2003). Neste contexto, o cereal se destaca como uma das principais culturas da agricultura, atingindo aumentos expressivos de produtividade, principalmente por meio do uso da transgenia (Garcia et al., 2013). Entretanto o uso de híbridos convencionais volta a ser uma opção de mercado devido à dificuldade de controle do milho voluntário resistente ao glyphosate $\left(\mathrm{RR}^{\circledR}\right)$ e as premiações oferecidas pelo grão convencional.

Dentre os fatores que podem limitar a utilização de híbridos convencionais destaca-se a ocorrência de deriva de glyphosate oriunda de lavouras adjacentes, ocasionada por reduções no diâmetro médio volumétrico das gotas e aumento da velocidade do vento (Cunha et al., 2017). Dessa forma a deposição do glyphosate pode reduzir às atividades enzimáticas e metabólicas, prejudicando sistematicamente os processos de síntese de compostos orgânicos (Mackinnon et al., 2010; Sharma et al., 2012). Entretanto, com aplicações de até 25,92 $\mathrm{g} \mathrm{ha}^{-1}$ e.a. de glyphosate, não foram observadas reduções nas características morfológicas e componentes de produtividade do milho (Felisberto et al., 2016).

O glyphosate é um herbicida sistêmico de amplo espectro de ação, transcolado via xilema e floema, sua ação leva a inibição da enzima EPSPS responsável por catalisar a condensação do ácido chiquímico (Coutinho e Mazo, 2005; Means e Kremer, 2007; Olesen e Cedergreen, 2010).
Com a inibição da rota do chiquimato ocorrerá redução da síntese de triptofano, este aminoácido é precursor da síntese do AIA que por sua vez promove a síntese de giberelinas, assim os processos de divisão e alongamento celular possivelmente serão inibidos (Carvalho et al., 2015; Taiz et al., 2017). Adicionalmente, o glyphosate pode afetar funções importantes como a síntese de clorofila e proteínas ocasionando redução na fotossíntese líquida (Magalhães et al., 2001; Carvalho et al., 2015).

Os eventos acima descritos retratam os efeitos causados por altas doses do herbicida no aparato fisiológico das plantas de milho, entretanto quando se trata de baixas doses os efeitos podem ser mais sutis, porém de grande impacto fisiológico e econômico. Um dos efeitos promovidos pelo glyphosate é a imobilização de $\mathrm{Cu}, \mathrm{Fe}, \mathrm{Mn}$ e $\mathrm{Zn}$, devido a propriedade quelante do herbicida ocasionada por sua ionização a um ânion fosfônico, com consequente redução nas atividades enzimáticas da planta (Zobiole et al., 2010; Craigie, 2011). Entretanto a duração destes sintomas é dependente da habilidade das plantas de repor os níveis adequados destes elementos através da absorção radicular ou foliar (Khan et al., 2012; Ferreira et al., 2013).

Dessa forma baixas doses de glyphosate na cultura do milho convencional pode comprometer o crescimento e o acúmulo de massa tanto das raízes quanto da parte aérea. Além disso a imobilização de micronutrientes que atuam como cofatores enzimáticos pode contribuir na redução da fotossíntese liquida comprometendo a síntese de fotossintatos e o desenvolvimento da cultura (Zobiole et al., 2010; Khan et al., 2012). Mesmo não causando a morte das plantas, diminutas doses 
podem causar danos parciais ao aparato fisiológico e morfológico, proporcionando danos que podem levar a grandes perdas de produtividade.

Dessa forma, uma alternativa de mitigar os efeitos deletérios ocasionado pela deriva de glyphosate é a utilização dos bioestimulantes. Dentre os bioestimulantes utilizados na agricultura estão os derivados da alga Ascophyllum nodosum, originária dos mares do leste canadense e noroeste europeu (Wally et al., 2013). Em função de sua composição (hormônios vegetais, aminoácidos, polissacarídeos, ácidos graxos, esteroides e poliaminas, além de macro e micronutrientes) são atribuídos a estes produtos funções nutricionais e enzimáticas (Mackinnon et al., 2010; Sharma et al., 2014). Além disso podem apresentar ação anti-estressante ocasionada pelo manutenção e acréscimo no conteúdo de clorofila, efeito relacionado às betaínas por reduzirem a fotodegradação dos pigmentos fotossintetizantes (Zobiole et al., 2010). Entretanto, ainda não há um consenso entre produtores e pesquisadores em relação a suas implicações fisiológicas.

Mediante ao exposto objetivo do trabalho foi avaliar o efeito de baixas doses de glyphosate nas características biométricas e na fotossíntese líquida de plantas de milho convencional, além de verificar o potencial do bioestimulante em reverter os efeitos da aplicação do herbicida.

\section{Material e métodos}

$\mathrm{O}$ experimento foi instalado em casa de vegetação na Universidade de Rio Verde - UniRV $\left(17^{\circ} 78^{\prime} 53,03^{\prime \prime}\right.$ de latitude sul e $50^{\circ} 96^{\prime} 48,69^{\prime \prime}$ de longitude oeste e altitude de $780 \mathrm{~m}$ ) no município de Rio Verde (GO). O período de condução do experimento foi de setembro a novembro de 2017.

Para o preenchimento dos vasos, foi coletado solo de uma área nativa do Cerrado, o qual fora classificado como Latossolo vermelho. Uma amostra do material coletado foi analisada e revelou as seguintes características químicas e físicas: $\mathrm{pH} \mathrm{em} \mathrm{CaCl}_{2}: 4,9$; $\mathrm{Ca}: 0,13 \mathrm{cmol}_{\mathrm{c}} \mathrm{dm}^{-3}$; Mg: $0,19 \mathrm{cmol}_{\mathrm{c}} \mathrm{dm}^{-3} ; \mathrm{Al}: 0,79 \mathrm{cmol}_{\mathrm{c}} \mathrm{dm}^{-3} ; \mathrm{H}+\mathrm{Al}$ :
$3,8 \mathrm{cmol}_{\mathrm{c}} \mathrm{dm}^{-3} ; \mathrm{K}: 0,3 \mathrm{cmol}_{\mathrm{c}} \mathrm{dm}^{-3} ; \mathrm{P}: 1,4 \mathrm{mg} \mathrm{dm}^{-3}$; CTC: $4,48 \mathrm{cmol}_{\mathrm{c}} \mathrm{dm}^{-3}, \mathrm{~V}: 13,52 \%$, argila $610 \mathrm{~g} \mathrm{~kg}^{-1}$, silte $70 \mathrm{~g} \mathrm{~kg}^{-1} \mathrm{e}$ areia $320 \mathrm{~g} \mathrm{~kg}^{-1}$.

Com base nos resultados da análise química do solo, foi realizada a calagem para a correção da acidez do solo, utilizando-se o calcário dolomítico finamente moído (PRNT=100\%), cuja composição apresentou $34,5 \%$ de óxido de cálcio e $18 \%$ de óxido de magnésio em quantidade necessária para elevar a saturação por bases a $65 \%$, permanecendo o solo incubado por 30 dias. A adubação de plantio foi realizada com o equivalente a $60 \mathrm{~kg} \mathrm{ha}^{-1} \mathrm{de}$ $\mathrm{K}_{2} 0$ e $80 \mathrm{~kg} \mathrm{ha}^{-1}$ de $\mathrm{P}_{2} \mathrm{O}_{5}$, efetuando-se a aplicação de 50 e $222 \mathrm{mg} \mathrm{dm}^{-3}$ de cloreto de potássio e superfosfato simples por unidade experimental, respectivamente.

As unidades experimentais foram constituídas por vasos de polietileno com capacidade para $8 \mathrm{dm}^{3}$. A irrigação foi realizada diariamente de forma localizada mantendo-se a umidade do solo equivalente a $60 \%$ do volume total de poros, sendo que o volume de água a aplicar foi estimado com base na densidade aparente (Dap) e no volume de poros $(\mathrm{Vp})$.

Foram semeadas cinco sementes de milho por vaso no dia 18/09/2017, empregando-se o híbrido convencional $30 \mathrm{~F} 53^{\circledR}$. Aos sete dias após a emergência foi realizado o desbaste deixando-se uma plântula por vaso.

O experimento foi instalado no delineamento inteiramente casualizados com quatro repetições, sendo empregados dez tratamentos. Foi adotado o arranjo fatorial ( 5 X 2), sendo que o primeiro fator correspondeu a sub - doses crescentes de glyphosate $\left(0 ; 10 ; 20 ; 30 ; 40\right.$ g e.a. ha $\left.{ }^{-1}\right)$ e o segundo fator a ausência e presença de bioestimulante em pós-emergência do milho. O bioestimulante utilizado no experimento foi o Bonder ${ }^{\circledR}\left(\mathrm{N}: 11,1 \mathrm{~g} \mathrm{~L}^{-1}\right.$; P: 22,2 g L L$^{-1}$; S: 11,1 g L-1; Carbono Orgânico: $90 \mathrm{~g} \mathrm{~L}^{-1}$, Alltech Crop Sience), o qual foi aplicado na dose de 3 L p.c ha ${ }^{-1}$. Para as aplicações de glyphosate, foi utilizado o produto comercial Roundup Transorb ${ }^{\circledR}$ (Concentração $580 \mathrm{~g} \mathrm{~L}^{-1}$, SC, Monsanto). 
Na ocasião das aplicações, os vasos foram alocados ao lado de fora da casa de vegetação. A aplicação simulando a deriva de glyphosate foi realizada quando as plantas de milho se encontravam em estádio $V_{5}$ (30 dias após a emergência das plantas). O bioestimulante foi aplicado sete dias após a aplicação do glyphosate.

Para as aplicações, foi utilizado pulverizador costal pressurizado por $\mathrm{CO}_{2}$ regulado a $300 \mathrm{kPa}$, munido de barra com seis pontas de pulverização tipo TT 110-02 leque duplo, posicionados na altura de $50 \mathrm{~cm}$ das plantas, proporcionou volume de calda equivalente a $200 \mathrm{~L} \mathrm{ha}^{-1}$. Após as aplicações, os vasos foram recolocados na casa de vegetação e foram irrigados apenas no dia seguinte, afim de assegurar a absorção foliar dos produtos aplicados. O horário e condições climáticas na ocasião das aplicações foram: 07:45 h e 10:25 h; velocidade do vento: 2 e $8,4 \mathrm{~km} \mathrm{~h}^{-1}$; temperatura: 23,4 e 25,8 ${ }^{\circ} \mathrm{C}$; umidade relativa 75 e $77 \%$, para as aplicações de glyphosate e bioestimulante, respectivamente.

Todas as avaliações foram realizadas aos sete dias após a aplicação do bioestimulante. As características avaliadas foram: Taxa fotossintética (A) em $\mu \mathrm{mol}$ $\mathrm{CO}_{2} \mathrm{~m}^{-2} \mathrm{~s}^{-1}$ (entre $9 \mathrm{~h}$ e $12 \mathrm{~h}$ tomando-se as medidas na terceira folha completamente expandida com fluxo de fótons fotossintéticamente ativos (FFFA) de $1000 \mu \mathrm{mol} \mathrm{m}{ }^{-2} \mathrm{~s}^{-1}$, com um analisador portátil de gases por infravermelho -IRGA, modelo CI-340 -CID Biosciences Inc., Camas, WA, USA), Índice SPAD (avaliou-se a terceira folha completamente expendida por meio do Clorofilômetro SPAD 502) e fitotoxicidade do herbicida a cultura, utilizando escala de notas onde $0 \%$ consistiam em plantas com ausência de injúrias e 100\% morte total das plantas (SBCPD, 1995).

Foi avaliado ainda o diâmetro do colmo (medindo-se na região abaixo da inserção do primeiro nó), altura plantas (por meio da medição do colo até a extremidade da última folha completamente expandida) e massa fresca de raiz e parte aérea (as plantas foram separadas em parte aérea e raiz exatamente no ponto de crescimento, com o auxílio de balança de precisão foi determinada a massa em gramas).

Os dados foram submetidos a análise estatística com emprego do teste $\mathrm{F}(\mathrm{p}<0,05)$, para constatar significância para os tratamentos. Uma vez constato efeito, foi empregada análise de regressão e teste de tukey $(p<0,05)$ para comparação das médias entre os tratamentos.

\section{Resultados e discussão}

Através dos resultados obtidos foi possível constatar que não houve interação entre as fontes de variação (Tabela 1). No entanto as baixas doses de glyphosate proporcionaram efeitos significativos para fitotoxidez, diâmetro de colmo, altura de planta, índice SPAD, matéria fresca da parte aérea além da taxa fotossintética (Tabela 1). Já a presença do bioestimulante proporcionou acréscimos para o diâmetro de colmo, altura de planta e índice SPAD (Figura 1), não sendo constatadas diferenças para as demais características.

Tabela 1. Significâncias das variáveis fitotoxidez (FITO), diâmetro de colmo (DC), altura de planta (AP), Índice SPAD (SPAD), massa fresca da parte aérea (MFPA), massa fresca da raiz (MFR) e taxa fotossintética (A) do ensaio com bioestimulante na redução de fitotoxidez de glyphosate na cultura do milho. Rio Verde (GO), 2017.

\begin{tabular}{cccccccc}
\hline FV & FITO & DC & AP & SPAD & MFPA & MFR & A \\
\hline Dose (D) & $* *$ & $\mathrm{~ns}$ & $* *$ & $* *$ & $*$ & $\mathrm{~ns}$ & $*$ \\
Bio (B) & $\mathrm{ns}$ & $* *$ & $* *$ & $* *$ & $\mathrm{~ns}$ & $\mathrm{~ns}$ & $\mathrm{~ns}$ \\
D x B & $\mathrm{ns}$ & $\mathrm{ns}$ & $\mathrm{ns}$ & $\mathrm{ns}$ & $\mathrm{ns}$ & $\mathrm{ns}$ & $\mathrm{ns}$ \\
CV $(\%)$ & 18,00 & 10,60 & 7,35 & 4,05 & 12,70 & 16,00 & 18,00 \\
\hline
\end{tabular}

**; * Significativo a 1 e $5 \%$ de probabilidade, respectivamente, pelo teste $\mathrm{F}$. 


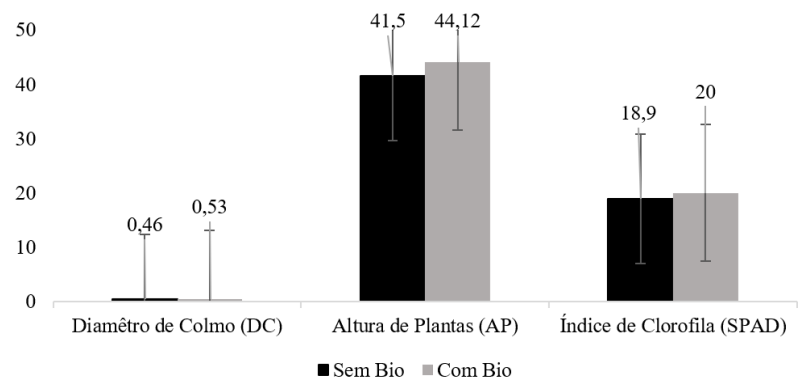

Figura 1. Diâmetro de colmo (cm), altura de planta $(\mathrm{cm})$ e índice SPAD das plantas de milho em função de ausência e presença de bioestimulante. Rio Verde GO, 2017. DC = Diâmetro de Colmo; $\mathrm{AP}=$ Altura de Plantas; SPAD = Índice de Clorofila.
Foi possível observar que a fitotoxidez foi influenciada pelas baixas doses de glyphosate, o aumento das doses promoveu acréscimos lineares para os valores da característica (Figura 2a). Este resultado indica a necessidade de empregar estratégias de redução de deriva e reversão do estresse fisiológico, visto que mesmo em doses baixas as plantas de milho apresentaram alta sensibilidade a molécula do glyphosate (Gheno et al., 2015). Uma das características do glyphosate é sua rápida translocação para as raízes promovendo sua subutilização (Zablotowicz e Reddy, 2007; Sharma et al., 2012)
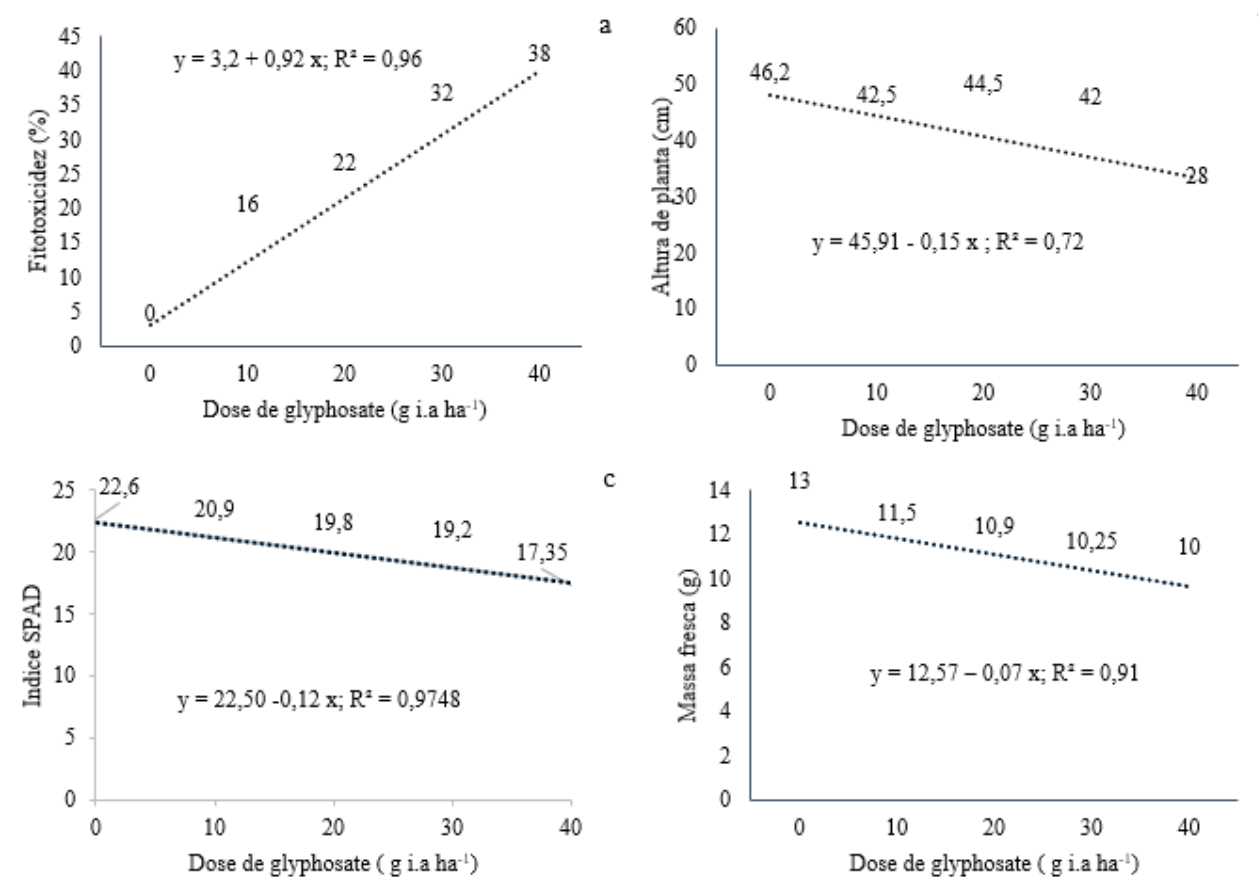

c
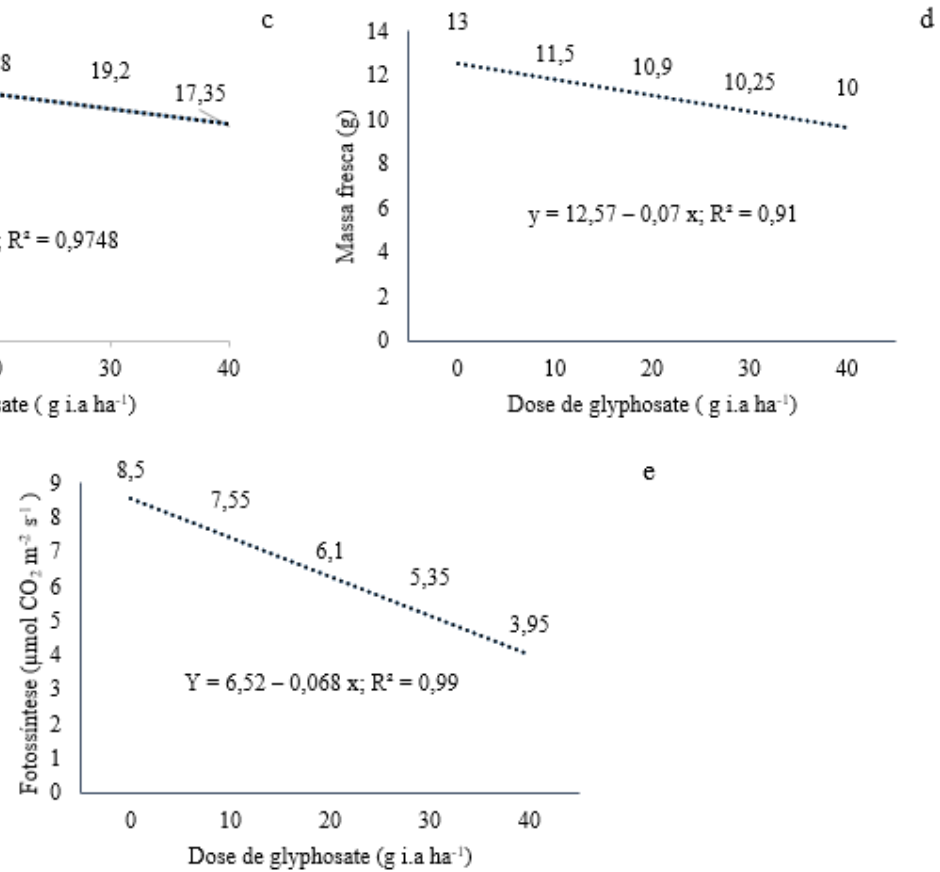

Figura 2. Fitotoxidez (a), altura das plantas (b), Índice SPAD (c), massa fresca da parte aérea (d) e taxa fotossintética (e) das plantas de milho em função das doses de glyphosate. Rio Verde GO, 2017. 
e consequentemente dificultando o processo de absorção de água e nutrientes, prolongando os sintomas de fitointoxicação.

A aplicação do bioestimulante não proporcionou efeito reparador a fitointoxicação das plantas de milho, refutando a hipótese do estudo (Tabela 1). Dessa forma acredita-se que em função da rápida translocação o glyphosate tenha causado a imobilização de altas concentrações de $\mathrm{Cu}, \mathrm{Fe}$, Mn e Zn (Reddy et al., 2004; Coutinho e Mazo, 2005; Ferreira et al., 2013) além disso o processo de biodegradação da molécula tenha resultado no acúmulo altas taxas de ácido aminometilfosfônico (AMPA) (Merotto et al., 2015) acentuando os sintomas de fitotoxidez.

Mesmo com os acréscimos dos sintomas de fitointoxicação o diâmetro do colmo das plantas de milho não foi influenciado pelas doses de glyphosate. (Tabela 1). Isto indica que as doses empregadas não foram suficientes para promover paralisação da divisão e alongamento celular, e que o acúmulo de AMPA não tenha sido suficiente para reduzir o acúmulo de biomassa, contrariando o relatado por Mackinnon et al. (2010). Adicionalmente, acredita-se que o intervalo entre a ocorrência da deriva e as avaliações não foi suficiente para que o efeito do herbicida pudesse ser expressado nesta característica.

Por outro lado, o bioestimulante incrementou o diâmetro de colmo, visto que sua aplicação proporcionou aumento de $13 \%$ no valor da característica (Figura 1). Este resultado contraria o encontrado por Gazola et al. (2014), estes autores concluíram que a aplicação foliar de bioestimulante a base de aminoácidos não proporcionou incrementos no diâmetro do colmo e no rendimento de grãos da cultura do milho.

O aumento das doses de glyphosate proporcionaram reduções lineares em altura de planta (Figura 2b). Isto ocorre pois com a inibição da EPSPS ocorrerá subutilização da rota do ácido chiquímico e consequentemente reduções na síntese de ácido indolilacético (IAA) e giberelinas
(GAA), responsáveis pela divisão e alongamento celular (Sharma et al., 2012; Taiz et al., 2017) justificando o resultado encontrado.

Por outro lado, os tratamentos com bioestimulante proporcionam plantas de maior porte (Figura 1). O bioestimulante possui traços de auxinas, citocininas e giberelinas, que em proporção adequada, tendem a otimizar a transmissão dos sinais químicos além da divisão e alongamento celular, proporcionando aumento de resistência a estresse biótico e abiótico. Dessa forma com do aumento da biossíntese de clorofila, pela adição dos bioestimulantes, ocorrerá incrementos na taxa fotossintética, tendo como consequência maior síntese de metabólitos e plantas de maior porte (Gomes et al., 2011; Khan et al., 2012).

$O$ índice SPAD apresentou a mesma tendência das variáveis avaliadas anteriormente, com superioridade dos tratamentos que receberam a aplicação do bioestimulante (Figura 1). Além disso ocorreram reduções lineares do índice SPAD com o aumento da dose de glyphosate (Figura 2c). Com a ionização do glyphosate a um ânion fosfônico, possivelmente ocorreu a imobilização importantes cofatores enzimáticos da biossíntese de clorofila (Zobiole et al., 2010; Khan et al., 2012; Silva et al., 2014), além disso o acúmulo de AMPA, mencionado anteriormente, pode ter contribuído para reduções nos valores da característica (Reddy et al., 2004; Beckie, 2011; Carvalho et al., 2015).

Adicionalmente o acúmulo de AMPA aliado à redução do índice SPAD ocasionados pela aplicação do glyphosate contribuíram para redução da produção de biomassa seca da plântula (Figura 2d), reforçando a necessidade de adoção de estratégias que visem beneficiar o desenvolvimento inicial das plântulas. Assim conforme o descrito por Andrade et al. (2018) o uso de bioestimulante, derivado de $A$. nodosum no tratamento de sementes de milho, proporcionou incrementos na massa seca e fresca da parte aérea, altura de plântulas e 
área foliar, e consequentemente maior potencial produtivo.

A taxa fotossintética e o índice SPAD apresentaram decréscimos proporcionais às doses de glyphosate aplicadas (Figuras 2e e 2c). Esses resultados estão de acordo com relatos feitos por agricultores, mostrando que alguns híbridos de milho são visualmente injuriados pelo herbicida. Este decréscimo da taxa fotossintética e do índice SPAD ocorre provavelmente pelo dano ao cloroplasto (Reddy et al., 2004) e pela imobilização de cátions como $\mathrm{Mg}$ e $\mathrm{Mn}$, requeridos para a formação de clorofila e para etapas da fotossíntese, respectivamente (Taiz et al., 2017).

O decréscimo da fotossíntese liquida corrobora com os resultados do índice SPAD, altura de plantas, fitotoxicidade e massa fresca de parte aérea. Visto que a redução da taxa fotossintética proporcionará menor altura de planta e menor produção de biomassa fresca da parte aérea, e, possivelmente, da biomassa total. De acordo com Albrecht et al. (2011), qualquer substância química que induz a alterações no metabolismo da folha, afeta a síntese metabólitos e a atividade das enzimas do ciclo de Calvin. Como a biomassa total das plantas dependem da energia da fotossíntese para sintetizar compostos carbônicos (Taiz et al., 2017), diminuições na assimilação do $\mathrm{CO}_{2}$ levam à redução na biomassa e no acúmulo de carboidratos (Magalhães Filho et al., 2008), resultando em plantas com menor potencial produtivo.

\section{Conclusões}

A maior dose de glyphosate proporcionou o maior sintoma visual de fitointoxicação, bem como reduções na altura de plantas, índice SPAD e taxa fotossintética. A presença de bioestimulante proporcionou acréscimos em diâmetro de colmo, altura de plantas e índice SPAD. Entretanto não foi constatado o efeito reparador do bioestimulante aos efeitos deletérios do glyphosate em plantas de milho convencional.

\section{Referências}

Albrecht, L.P.; Braccini, A.L.; Scapim, C.A.; Ávila, M.R.; Albrecht, A.J.P.; Ricci, T.T. Manejo de biorregulador nos componentes de produção e desempenho das plantas de soja. Bioscience Journal, v.27, n.6, p.865-876, 2011.

Andrade, C.L.L.; Silva, A.G.; Cantão, V.C.G.; Mello, G.B.; Siqueira, G.G.C.; Rodrigues, R.L.S. Performance of maize seedlings using biostimulant in seed treatment. Científica, v.46, n.3, p.274-282, 2018.

Beckie, H.J. Herbicide-resistant weed management: focus on glyphosate. Pest Management Science, v.67, n.9, p.1037-1048, 2011.

Carvalho, L.B.; Pereira, M.; Borges, P.; Silva, F.J.; Costa, F. Resposta diferencial das culturas de milho RR e soja RR à exposição a glyphosate e adubação fosfatada. Planta Daninha, v.33, n.4, p.751, 2015.

Coutinho, C.F.B.; Mazo, L.H. Metallic complex with glyphosate: a review. Quimica Nova, v.28, n.6, p.1038-1045, 2005.

Cunha, J.P.A.R.; Alves, G.S.; Marques, R.S. Tensão superficial, potencial hidrogeniônico e condutividade elétrica de caldas de produtos fitossanitários e adjuvantes. Revista Ciência Agronômica, v.48, n.2, p.261-270, 2017.

Craigie, J.S. Seaweed extract stimuli in plant science and agriculture. Journal of Applied Phycology, v.23, n.3, p.371-393, 2011.

Garcia, C.M.D.P.; Andreotti, M.; Carvalho, M.T.F.M.; Buzetti, S.; Souza Celestrino, T.; Mascarenhas Lopes, K.S. Desempenho agronômico da cultura do milho e espécies forrageiras em sistema de Integração Lavoura-Pecuária no Cerrado. Ciência Rural, v.43, n.4, p.589-595, 2013.

Felisberto, P.A.; Timossi, P.C.; Felisberto, G.; Ramos, A.R. Subdoses de glyphosate não reduzem a produtividade da cultura do milho. Revista 
Brasileira de Herbicidas, v.15, n.3, p.290-296, 2016.

Ferreira, L.R.; Silva, A.G.; Simon, G.A.; Teixeira, I.R.; Sena, M.P.D. Glyphosate em pós-emergência na soja Roundup Ready. Revista Brasileira de Herbicidas, v.12, n.2, p.151-161, 2013.

Gazola, D.; Zucareli, C.; Silva, R.R.; Fonseca, I.C.D.B. Aplicação foliar de aminoácidos e adubação nitrogenada de cobertura na cultura do milho safrinha. Revista Brasileira de Engenharia Agrícola e Ambiental-Agriambi, v.18, n.7, p.700-707, 2014.

Gheno, E.A.; Oliveira Junior, R.S.; Constantin, J.; Biffe, D.F.; Pereira, G.B.; Braz, F.A.R.; Gonçalves, V.D.B. Atividade residual de herbicidas aplicados em diferentes modalidades no algodoeiro sobre milho cultivado em sucessão. Revista Brasileira de Milho e Sorgo, v. 14, n.3, p.335-345, 2015.

Gomes, K.R.; Amorim, A.V.; Ferreira, F.J.; Francisco Filho, L.A.; Lacerda, C.F.; GomesFilho, E. Respostas de crescimento e fisiologia do milho submetido a estresse salino com diferentes espaçamentos de cultivo. Revista Brasileira de Engenharia Agrícola e Ambiental-Agriambi, v.15, n.4, p.365-370, 2011.

Khan, A.S.; Ahmad, B.; Jaskani, M.J.; Ahmad, R.; Malik, A.U. Foliar application of mixture of amino acids and seaweed Ascophylum nodosum extract improve growth and physicochemical properties of grapes. International Journal of Agriculture and Biology, v.14, n.3, p.383-388, 2012.

Mackinnon, S.L.; Hiltz, D.; Ugarte, R.; Craft, C.A. Improved methods of analysis for betaines in Ascophyllum nodosum and its commercial seaweed extracts. Journal of Applied Phycology, v.22, n.4, p.489-494, 2010.

Magalhaes Filho, R.; Rios do Amaral, L.; Fávero São Pedro Machado, D.; Medina, C.L.; Caruso, M.E. Deficiência hídrica, trocas gasosas e crescimento de raízes em laranjeira 'Valência' sobre dois tipos de porta-enxerto. Bragantia, v.67, n.1, p.75-82, 2008.

Magalhães, P.C.; Silva, J.B.; Durães, F.O.M.; Karam, D.; Ribeiro, L.S. Efeito de doses reduzidas de glyphosate e paraquat simulando deriva na cultura do milho. Planta Daninha, v.19, n.2, p.247-253, 2001.

Means, N.E.; Kremer, R.J. Influence of soil moisture on root colonization of glyphosatetreated soybean by Fusarium species. Communications in Soil Science and Plant Analysis, v.38, n.1314, p.1713-1720, 2007.

Merotto, A.; Wagner, J.; Meneguzzi, C. Effects of Glyphosate and foliar application of micronutrients in transgenic soybean. Bioscience Journal, v.31, n.2, p.499-508, 2015.

Olesen, C.F.; Cedergreen, N. Glyphosate uncouples gas exchange and chlorophyll fluorescence. Pest Management Science, v.66, n.5, p.536-542, 2010.

Reddy, K.N.; Rimando, A.M.; Duke, S.O. Aminomethylphosphonic acid, a metabolite of glyphosate, causes injury in glyphosate-treated, glyphosate-resistant soybean. Journal of Agricultural and Food Chemistry, v.52, n.16, p.5139-5143, 2004.

Ribas, P.M. Sorgo: introdução e importância econômica. Sete Lagoas: Embrapa Milho e Sorgo, 2003. 16p. (Embrapa Milho e Sorgo. Documentos, 26).

SBCPD - Sociedade Brasileira da Ciência das Plantas Daninhas. Procedimentos para instalação, avaliação e análise de experimentos com herbicidas. Londrina: SBCPD, 1995. 42 p.

Silva, F.B.; Costa, A.C.; Alves, R.R.P.; Megguer, C.A. Chlorophyll fluorescence as an indicator of cellular damage by glyphosate herbicide in Raphanus sativus L. plants. American Journal of Science, v.5, n.16, p.2509-2519, 2014.

Sharma, H.S.; Fleming, C.; Selby, C.; Rao, J.R.; Martin, T. Plant biostimulants: a review on the processing of macroalgae and use of extracts for crop management to reduce abiotic and biotic 
stresses. Journal of Applied Phycology, v.26, n.1, p.465-490, 2014.

Sharma, S.H.S.; Lyons, G.; Mcroberts, C.; Mccall, D.; Carmichael, E.; Andrews, F.; et al. Biostimulant activity of brown seaweed species from Strangford Lough: compositional analyses of polysaccharides and bioassay of extracts using mung bean (Vigno mungo L.) and pak choi (Brassica rapa chinensis L.). Journal of Applied Phycology, v.24, n.5, p.1081-1091, 2012.

Taiz, L.; Zeiger, E.; Moller, I.M.; Murphy, A. Fisiologia e desenvolvimento vegetal. 6.ed. Porto Alegre: Artmed, 2017. 888p.

Wally, O.S.D.; Critchley, A.T.; Hiltz, D.; Craigie, J.S.; Han, X.; Zaharia, L.I.; et al. Regulation of phytohormone biosynthesis and accumulation in Arabidopsis following treatment with commercial extract from the marine macroalga Ascophyllum nodosum. Journal of Plant Growth Regulation, v.32, n.2, p.324-339, 2013.

Zablotowicz, R.M.; Reddy, K.N. Nitrogenase activity, nitrogen content, and yield responses to glyphosate in glyphosate-resistant soybean. Crop Protection (Guildford, Surrey), v.26, n.3, p.370-376, 2007.

Zobiole, L.H.S.; Oliveira Junior, R.S.; Huber, D.M.; Constantin, J.; Castro, C.; Oliveira, F.A.; Oliveira, A. Glyphosate reduces shoot concentration of mineral nutrients in glyphosate resistant soybeans. Plant and Soil, v.328, n.1, p.57-69, 2010. 